\title{
Epitaxially stabilized iridium spinel oxide without cations in the tetrahedral site
}

Hiromichi Kuriyama, ${ }^{1,2}$ Jobu Matsuno, ${ }^{2}$ Seiji Niitaka, ${ }^{2}$ Masaya Uchida, ${ }^{2}$ Daisuke Hashizume, ${ }^{2}$ Aiko Nakao, ${ }^{2}$ Kunihisa Sugimoto, ${ }^{3}$ Hiroyuki Ohsumi, ${ }^{4}$ Masaki Takata, $^{4}$ and Hidenori Takagi ${ }^{1,2}$

\footnotetext{
${ }^{1}$ Department of Advanced Materials, University of Tokyo, 5-1-5 Kashiwanoha, Kashiwa, Chiba 277-8561, Japan

${ }^{2}$ Advanced Science Institute, RIKEN, 2-1 Hirosawa, Wako, Saitama 351-0198, Japan

${ }^{3}$ Japan Synchrotron Radiation Research Institute, SPring-8, 1-1-1 Kouto, Sayo-cho, Sayo-gun, Hyogo 679-5198, Japan

${ }^{4}$ RIKEN, SPring-8 Center, 1-1-1 Kouto, Sayo-cho, Sayo-gun, Hyogo 679-5148, Japan
}

Single-crystalline thin film of an iridium dioxide polymorph $\operatorname{Ir}_{2} \mathrm{O}_{4}$ has been fabricated by the pulsed laser deposition of $\mathrm{Li}_{x} \mathrm{Ir}_{2} \mathrm{O}_{4}$ precursor and the subsequent Li-deintercalation using soft chemistry. $\operatorname{Ir}_{2} \mathrm{O}_{4}$ crystallizes in a spinel $\left(A B_{2} \mathrm{O}_{4}\right)$ without $A$ cations in the tetrahedral site, which is isostructural to $\lambda-\mathrm{MnO}_{2}$. Ir ions form a pyrochlore sublattice, which is known to give rise to a strong geometrical frustration. This Ir spinel was found to be a narrow gap insulator, in remarkable contrast to the metallic ground state of rutile-type $\mathrm{IrO}_{2}$. We argue that an interplay of strong spin-orbit coupling and a Coulomb repulsion gives rise to an insulating ground state as in a layered perovskite $\mathrm{Sr}_{2} \mathrm{IrO}_{4}$. 
$5 d$ transition metal oxides (TMOs) with an odd number of $d$-electrons per transition metal ion are generally believed to be a wide band metal due to the spatially extended character of outermost $5 d$ orbitals. Recent discovery of an unconventional Mott insulating state in a layered perovskite $\mathrm{Sr}_{2} \mathrm{IrO}_{4}$, however, has shown that an interplay of a strong spin-orbit coupling (SOC) inherent to heavy $5 d$ elements and a Coulomb repulsion $U$ cannot be ignored

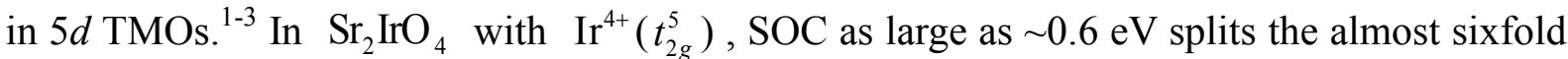
degenerate $t_{2 \mathrm{~g}}$ bands into effective total angular momentum $J_{\text {eff }}=1 / 2\left(J_{1 / 2}\right)$ and $3 / 2$ $\left(J_{3 / 2}\right)$ states, yielding a half-filled $J_{1 / 2}$ band. The presence of the narrow half filled band gives rise to an antiferromagnetic Mott insulating state with a modest Coulomb $U \sim 0.5 \mathrm{eV}$. Such SOC-induced Mott insulator is now attracting great attention as an exotic state of matter.

If the uniqueness of $\operatorname{Ir}^{4+}$ is coupled with a special topology of lattice, such as geometrically frustrated lattice, even more exotic ground state such as correlated topological insulator may be anticipated., ${ }^{4,5}$ We put our attention on $A B_{2} \mathrm{O}_{4}$-type normal spinel compound, where $B$ cations in the octahedral site comprise pyrochlore sublattice with corner sharing tetrahedra as shown in Fig. 1. The triangular-lattice-based geometry of pyrochlore lattice is known to produce incompatibility of interactions between the neighboring magnetic $B$ ions, called geometrical frustration. To accommodate $\operatorname{Ir}^{4+}$ in the spinel $B$-sites, we have to develop a spinel $\square \mathrm{Ir}_{2} \mathrm{O}_{4}$ without $A$ cations in the tetrahedral site, isostructural to $\lambda-\mathrm{MnO}_{2}$, for the sake of charge neutrality. ${ }^{6}$

We attempted to grow $\lambda-\mathrm{MnO}_{2}$-type $\mathrm{Ir}_{2} \mathrm{O}_{4}$ by employing thin film technique and soft chemical method. So far, however, spinel oxide with Ir has not been synthesized yet in the bulk form. The only example of spinel-type Ir oxide known is $\mathrm{ZnIr}_{2} \mathrm{O}_{4}$, which was stabilized by utilizing thin film technique. ${ }^{7}$ Our early attempts to grow $\operatorname{Ir}_{2} \mathrm{O}_{4}$ directly on substrates were not successful. Since $\lambda-\mathrm{MnO}_{2}$ was reported to be synthesized by a Li-deintercalation from $\mathrm{LiMn}_{2} \mathrm{O}_{4}$ spinel, ${ }^{6}$ we took essentially the same approach for $\operatorname{Ir}_{2} \mathrm{O}_{4}$. In this work, by deintercalating $\mathrm{Li}$ from epitaxially grown $\mathrm{Li}_{x} \mathrm{Ir}_{2} \mathrm{O}_{4}$ films, $\mathrm{Ir}_{2} \mathrm{O}_{4}$ spinel without $A$ cations was synthesized. This unique form of $\mathrm{Ir}_{2} \mathrm{O}_{4}$ was found to be an insulator in remarkable 
contrast to the metallic ground state of rutile-type $\mathrm{IrO}_{2}$.

Epitaxial thin film of $\mathrm{Li}_{x} \mathrm{Ir}_{2} \mathrm{O}_{4}$ precursor was grown on $\mathrm{LiNbO}_{3}(0001)$ substrate. Films with 70-100 nm thickness were deposited by pulsed laser deposition (PLD) using a $\mathrm{KrF}$ excimer laser $(\lambda=248 \mathrm{~nm})$ at $5 \mathrm{~Hz}$ with a fluency of $\sim 3 \mathrm{~J} / \mathrm{cm}^{2}$ on the target surface. An oxygen partial pressure and a deposition temperature of $10 \mathrm{~Pa}$ and $923 \mathrm{~K}$, respectively, were used for the optimized thin film growth in this study. A Li-rich target with a composition of $\mathrm{Li} / \mathrm{Ir} \sim 1$ was used to compensate the volatilization of Li during the deposition. The target was prepared by a solid-state reaction. The mixture of $\mathrm{Li}_{2} \mathrm{CO}_{3}$ and $\mathrm{IrO}_{2}$ was calcined in air at $923 \mathrm{~K}$ for $12 \mathrm{~h}$. The product was finely ground and sintered in air at $1023 \mathrm{~K}$ for $48 \mathrm{~h}$. The obtained target was found to be a mixture of $\mathrm{Li}_{2} \mathrm{IrO}_{3}$ and $\mathrm{IrO}_{2}$. The crystal structures were characterized by various $\mathrm{x}$-ray diffraction (XRD) measurements using $\mathrm{Cu} K \alpha$ radiation. The composition of films was checked by x-ray photoelectron spectroscopy (XPS).

In XRD measurement of $2 \theta-\omega$ scan on obtained films shown in Fig. 2(a), the presence of a single phase of highly oriented Li-Ir-O can be recognized. The full width at half maximum of rocking curve of $\omega$ scan was estimated to be $0.07^{\circ}-0.1^{\circ}$ for the peak at the lowest $2 \theta$ angle, indicating a good crystallinity of the Li-Ir-O. The detailed $\mathrm{x}$-ray data indicate that $\mathrm{Li}_{x} \mathrm{Ir}_{2} \mathrm{O}_{4}$ spinel is formed as naively expected from the matching with the trigonal $\mathrm{LiNbO}_{3}$ (0001) surface. The Li/Ir composition in Li-Ir-O films was estimated to be $0.3-0.5$ by XPS measurement, excluding the possibility of known Li-Ir-O compounds such as $\mathrm{Li}_{8} \mathrm{IrO}_{6}$ and $\mathrm{Li}_{2} \mathrm{IrO}_{3}$, all with $\mathrm{Li}$ rich composition. The largest $d$ value $d_{\max }=4.95 \AA$, evaluated from lowest $2 \theta$ peak at $17.91^{\circ}$, was found to be comparable to the value of $d_{111}$ for spinel. The asymmetric reflections such as (226), (551) and (153) indexed by assuming spinel structure were observed as shown in Fig. 2(d)-(f). The reciprocal mapping around (226) exhibits that the epitaxial relationship between film and substrate is $[11 \overline{2}]$ spinel $\mathrm{Li}_{x} \mathrm{Ir}_{2} \mathrm{O}_{4} \|[11 \overline{2} 0]$ $\mathrm{LiNbO}_{3}$. This alignment should stabilize the interface with matching of oxygen-arrangement. To further justify the growth of spinel, we checked $(004 n)$ reflection by fabricating Li-Ir-O thin films on $\mathrm{MgO}(001)$ substrate, while $(00 l)$ reflections cannot be approached as long as 
films have (111)-orientation. The observation of (226), (551), (153) and (004n) peaks are fully consistent with reflection conditions of space group $F d \overline{3} m$ (No.227) for spinel; $h h l$ : $h+l=2 n, h k l: h+k, h+l, k+l=2 n$ and $00 l: l=4 n$. Considering the contrast of ionic charge and ionic radius, though the structural details are not known, it is highly likely that Ir occupies only the octahedral $B$ sites and that Li occupies only the tetrahedral $A$ sites of spinel.

We found that $\mathrm{Li}$ ions can be removed from $\mathrm{Li}_{x} \mathrm{Ir}_{2} \mathrm{O}_{4}$ by soft chemical reaction. $\mathrm{Li}$ ions were extracted by dipping $\mathrm{Li}_{x} \operatorname{Ir}_{2} \mathrm{O}_{4}$ film into $0.02 \mathrm{M} \mathrm{I} \mathrm{I}_{2}$ /acetonitrile solution at room temperature. The framework of structure was maintained after the Li-deintercalation which is evidenced by XRD pattern in Fig. 2(a). The small value of full width at half maximum of rocking curve of $\omega$ scan of $0.07^{\circ}-0.1^{\circ}$ for (111) reflection indicates that the crystallinity is maintained. Taking look at details, we notice that the peak positions of $(h h h)$ reflections shifted to higher $2 \theta \mathrm{s}$ [Fig. 2(b)], indicating that the cell volume was reduced. This reflects the deintercalation of Li. The XPS spectra of the same films clearly indicated the absence of Li $1 \mathrm{~s}$ peak within our resolution. From these results, we concluded that Li ions were entirely removed from the film without any degradation of crystallinity and that $\operatorname{Ir}_{2} \mathrm{O}_{4}$ spinel without $A$ cations is formed.

The electrical resistivity and optical conductivity spectrum of $\mathrm{Ir}_{2} \mathrm{O}_{4}$ film on $\mathrm{LiNbO}_{3}$ were measured. We found that $\mathrm{Ir}_{2} \mathrm{O}_{4}$ is a narrow gap insulator in remarkable contrast to the highly conductive metallic state of rutile-type $\mathrm{IrO}_{2}$. As shown in Fig. 3(a), $\mathrm{Ir}_{2} \mathrm{O}_{4}$ exhibits an insulating behavior with a relatively low resistivity of several milliohm centimeter at room temperature. An activation energy of $\sim 0.034 \mathrm{eV}$ was obtained from the resistivity data above $200 \mathrm{~K}$. The low resistivity and the low activation energy indicate that the energy gap of $\operatorname{Ir}_{2} \mathrm{O}_{4}$ is likely very small. The narrow energy gap is indeed evidenced by an optical conductivity spectrum $\sigma(\omega)$ measured at room temperature shown in Fig. 3(c). It is clear in Fig. 3(c) that a narrow charge gap is present below a peak $(\alpha)$ at $0.5 \mathrm{eV}$, and that the energy gap should be much smaller than $0.5 \mathrm{eV}$. These transport and spectroscopic studies indicate that $\operatorname{Ir}_{2} \mathrm{O}_{4}$ is a narrow gap insulator. 
The XPS measurement revealed that $\mathrm{Ir}_{2} \mathrm{O}_{4}$ is classified as a $t_{2 g}^{5}$ system with $\mathrm{Ir}$ in the octahedral $B$ site. The valence-band spectrum is composed of a broad structure ranging from 3 to $9 \mathrm{eV}$ and an intense peak at around $1.5 \mathrm{eV}$ [Fig. 3 (b)]. As observed in $\mathrm{ZnIr}_{2} \mathrm{O}_{4}{ }^{7}$, the former originates mainly from $\mathrm{O} 2 p$ states, while the latter is assigned to $\operatorname{Ir} 5 d$ states. Under the octahedral ligand field at the $B$ site, the $5 d$ states split into $t_{2 g}$ and $e_{g}$ manifolds separated by $\Delta_{\mathrm{LF}} \sim 3 \mathrm{eV}$. The observed band width of $\sim 3 \mathrm{eV}$ for $5 d$ states indicates the absence of the splitting $\Delta_{\mathrm{LF}}$ in the valence band: five $d$ electrons of $\operatorname{~Ir~}^{4+}$ are accommodated only to $t_{2 g}$ states with the low-spin $t_{2 g}^{5}$ configuration. Thus we concluded that $\operatorname{Ir}^{4+}$ takes the low-spin $t_{2 g}^{5}$ state in the octahedral $B$ site. $^{8}$

The detailed electronic structure of Ir 5d states are observed in optical conductivity $\sigma(\omega)$ [Fig. 3 (c)]. The spectral feature in $\sigma(\omega)$ can be decomposed into two parts below and above $\hbar \omega \simeq 2.5 \mathrm{eV}$. The high energy absorption is similar to those observed for $\mathrm{ZnIr}_{2}^{3+} \mathrm{O}_{4}$ with completely filled $t_{2 \mathrm{~g}}$ bands. ${ }^{7}$ We assign the absorption spectrum above $3 \mathrm{eV}$ to the interband transition from $t_{2 \mathrm{~g}}$ to empty $e_{\mathrm{g}}$, corresponding to the octahedral crystal field splitting $\Delta_{\mathrm{LF}}$. The two peaks, $\alpha$ and $\beta$, at low energies $\hbar \omega=0.5 \mathrm{eV}(\alpha)$ and $1.4 \mathrm{eV}(\beta)$ should be assigned to the intraband transition within $t_{2 \mathrm{~g}}$ manifolds, which is absent in $\mathrm{ZnIr}_{2} \mathrm{O}_{4}{ }^{7}$

The presence of two absorption peaks below $2 \mathrm{eV}$ means a complicated and multiple splitting of $t_{2 \mathrm{~g}}$ bands. The two peaks at low energies is strikingly in parallel with those observed in other $\mathrm{Ir}^{4+}$ insulator $\mathrm{Sr}_{2} \mathrm{IrO}_{4}$, very likely indicating that the strong SOC splits the $t_{2 \mathrm{~g}}$ manifolds into $J_{1 / 2}$ bands and $J_{3 / 2}$ bands as in $\mathrm{Sr}_{2} \mathrm{IrO}_{4} \cdot{ }^{1-3}$ The electronic structure and the optical transitions adapted from those of $\mathrm{Sr}_{2} \mathrm{IrO}_{4}$ are summarized in the schematics shown in Fig. 3(d). The peak $\alpha$ is assigned to a Mott-Hubbard transition within $J_{1 / 2}$ bands while the peak $\beta$ corresponds to a transition from filled $J_{3 / 2}$ bands to the upper Hubbard bands with $J_{1 / 2}$. The $\alpha$ peak energy of $0.5 \mathrm{eV}$, which is comparable to that of $\mathrm{Sr}_{2} \mathrm{IrO}_{4}$, indicates that even modest value of $U$ can open a Mott gap over a variety of $\mathrm{Ir}^{4+}$ oxides.

In summary, we have synthesized $\mathrm{Ir}_{2} \mathrm{O}_{4}$ spinel without $A$ cations via $\mathrm{Li}_{x} \mathrm{Ir}_{2} \mathrm{O}_{4}$ by 
utilizing PLD technique and soft chemical method. $\mathrm{Ir}_{2} \mathrm{O}_{4}$ is isostructural to $\lambda-\mathrm{MnO}_{2}$, which is composed of $\mathrm{Ir}^{4+}$-pyrochlore lattice. It was revealed, by the measurements of optical spectrum and electrical resistivity, that $\mathrm{Ir}_{2} \mathrm{O}_{4}$ is a narrow gap insulator. The insulating state is highly likely a SOC-assisted Mott insulator as in $\mathrm{Sr}_{2} \mathrm{IrO}_{4}$. To further explore the outcome of additional ingredients in this unique $\mathrm{Ir}^{4+}$ oxide, in particular the geometrical frustration, the measurement of magnetism is highly desired. The resonant x-ray technique using Ir L edge should be quite powerful probe for this.

This work was financially supported by Grant-in-Aid for Scientific Research (S) (19104008), Grant-in-Aid for Scientific Research on Priority Areas (19052008) and Grant-in Aid for JSPS Fellows.

${ }^{1}$ B. J. Kim, H. Jin, S. J. Moon, J.-Y. Kim, B.-G. Park, C. S. Leem, J. Yu, T. W. Noh, C. Kim, S.-J. Oh, J.-H. Park, V. Durairaj, G. Cao, and E. Rotenberg, Phys. Rev. Lett. 101, 076402 (2008).

${ }^{2}$ B. J. Kim, H. Ohsumi, T. Komesu, S. Sakai, T. Morita, H. Takagi, and T. Arima, Science 323, 1329 (2009).

${ }^{3}$ S. J. Moon, H. Jin, K.W. Kim, W. S. Choi, Y. S. Lee, J. Yu, G. Cao, A. Sumi, H. Funakubo, C. Bernhard, and T. W. Noh, Phys. Rev. Lett. 101, 226402 (2008).

${ }^{4}$ H.-M. Guo and M. Franz, Phys. Rev. Lett. 103, 206805 (2009).

${ }^{5}$ D. A. Pesin and L. Balents, arXiv:0907.2962 (unpublished).

${ }^{6}$ J. C. Hunter, J. Solid State Chem. 39, 142 (1981).

${ }^{7}$ M. Dekkers, G. Rijnders, and D. H. A. Blank, Appl. Phys. Lett. 90, 021903 (2007).

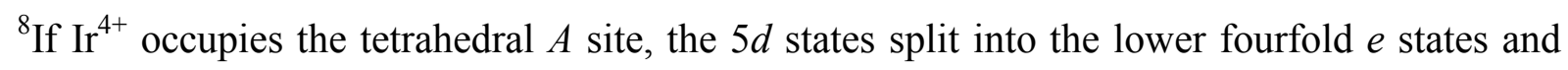
the higher sixfold $t_{2}$ states. Then five $d$ electrons of $\operatorname{Ir}^{4+}$ are inevitably distributed to both $e$ and $t_{2}$ states under any possible electron configuration such as $e^{4} t_{2}{ }^{1}$ or $e^{2} t_{2}{ }^{3}$. This is inconsistent with the absence of the ligand field splitting in the valence band spectrum and therefore we can eliminate the possibility that Ir occupies the $A$ site. 


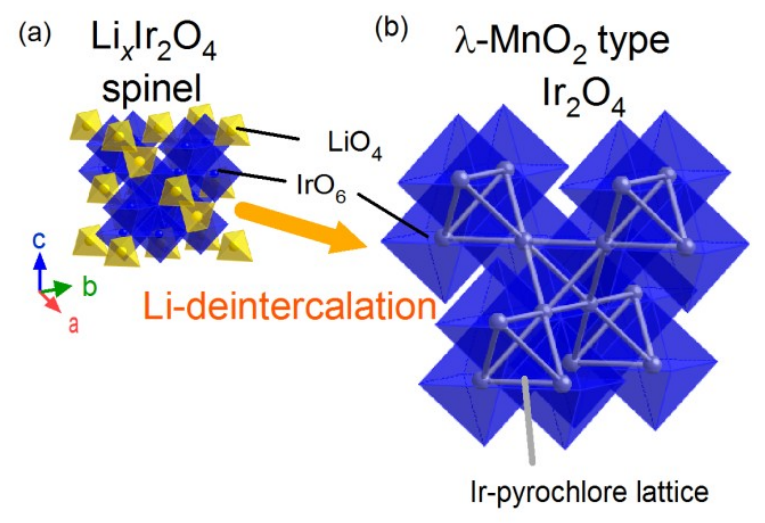

FIG. 1. (Color online) Crystal structures of spinel-type oxide (a) $\mathrm{LiIr}_{2} \mathrm{O}_{4}$ and (b) $\operatorname{Ir}_{2} \mathrm{O}_{4}$. The Ir-pyroclore sublattice is shown by the balls and the sticks in (b). $\mathrm{Ir}_{2} \mathrm{O}_{4}$ represents a Li-deintercalated analog of $\operatorname{Lirr}_{2} \mathrm{O}_{4}$ in this paper. 

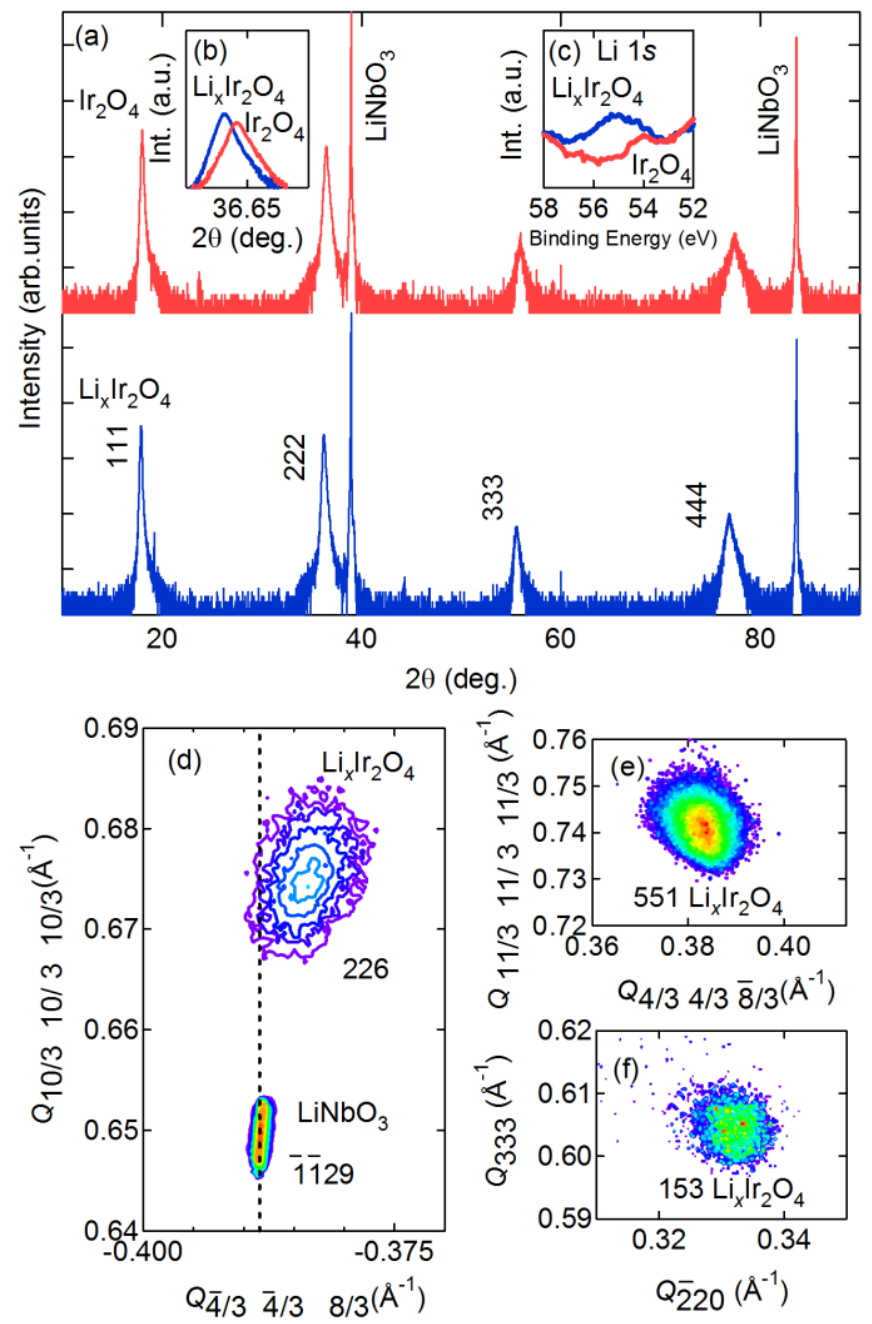

FIG. 2. (Color online) X-ray characterization of $\mathrm{Li}_{x} \mathrm{Ir}_{2} \mathrm{O}_{4}$ and $\mathrm{Ir}_{2} \mathrm{O}_{4}$ films on $\mathrm{LiNbO}_{3}$ (0001) substrate. (a) XRD patterns of $2 \theta-\omega$ scan over a wide range. (b) Enlarged view of (222) reflection. (c) XPS spectra of $\mathrm{Li} 1 s$ peak. Reciprocal space maps for $\mathrm{Li}_{x} \mathrm{Ir}_{2} \mathrm{O}_{4}$ thin film around (d) (226), (e) (551) and (f) (153). 

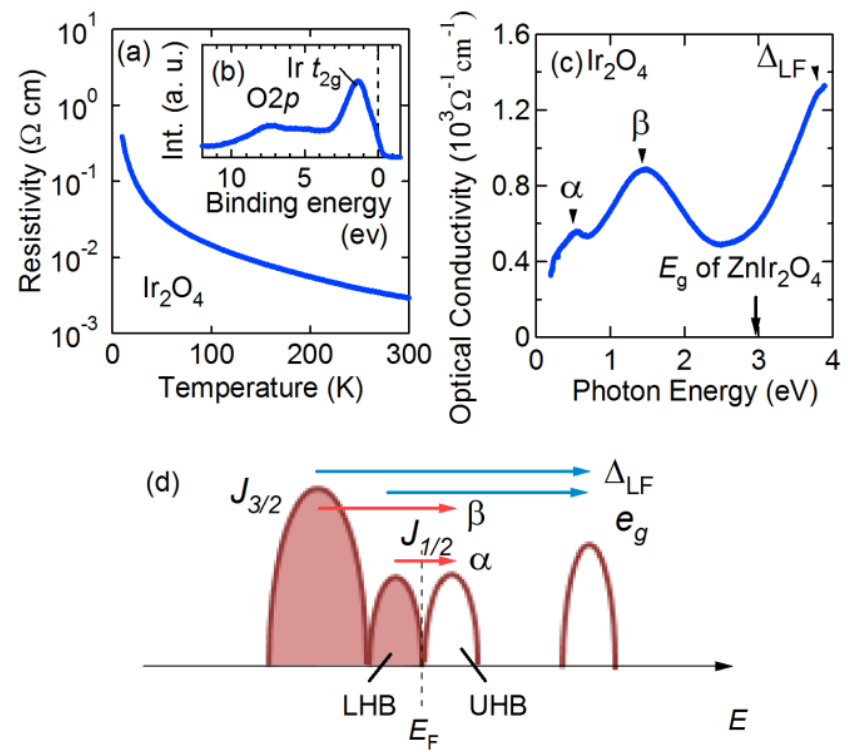

FIG. 3. (Color online) Transport and spectroscopic properties of $\operatorname{Ir}_{2} \mathrm{O}_{4}$ thin film. (a) Temperature dependence of electrical resistivity. (b) Valence-band x-ray photoelectron spectroscopy using Al $\mathrm{K \alpha}$ excitation source. (c) Optical conductivity measured at room temperature. (c) Schematic view of electronic structure for $\operatorname{Ir}_{2} \mathrm{O}_{4}$ based on Ref. 1. The position of band gap $\left(E_{\mathrm{g}} \sim 2.97 \mathrm{eV}\right.$ ) for $\mathrm{ZnIr}_{2}^{3+} \mathrm{O}_{4}$ (Ref. 7) is indicated by an arrow in (c) for comparison. 